\title{
Régime du cours inférieur de la Loire
}

\section{The regime of the lower Loire}

PAR

\author{
M. PARDÉ
}

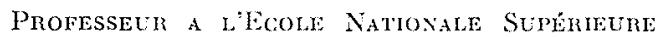
D'Electroteghidue et D'HydrauliQue me Grenomil:
EI

\author{
Direcreun des ports de Nantes ex SaInT-Nazalle,
} Ingéneun en Chef du Senvice spécial de la Lome

English synopsis p. 218

\section{1. - INTRODUCTION}

Parmi les grands fleuves français, la Loire est celui pour lequel on possède l'ensemble le plus complet de jaugeages modernes, du moins dans son cours inférieur.

Le Service Maritime de Nantes el de SaintNazaire, que j'ai l'honneur de diriger, est également chargé de la gestion du Service Spécial de la Loire depuis l'embouchure de la Vienne, le grand affluent de la rive gauche, et la limite de la mer. Il s'intéresse à la fois au régime des crues, ayant la responsabilité des levées de protection dans cette importante partie du cours du fleuve, et à celui des basses-eaux qui conditionne lexercice de la navigation dans la partie du fleuve aménagée à courant libre, il y a une trentaine d'années entre Angers et Nantes.

La tenue des eaux d'amont influence également le régime de la partie maritime, soit que les crues aient pour effet d'y provoquer des chasses salutaires, soit qu'au contraire des insuffisances estivales aient pour corollaire des remontées de vase très gênantes dans le port de Nantes.

C'est ce qui explique qu'une station d'observation de la tenue des eaux du fleuve ait été organisée depuis plus d'un siècle à un point du cours inférieur situé à Montjean, à une vingtaine de kilomètres en aval du confluent avec la Maine. Indépendamment de l'observation journalière de la hauteur des eaux à l'ćchelle (plus fréquente en temps de crue), de nombreux jaugeages y ont été pratiqués par flotteurs super- ficiels entre les années 1844 et 1909 ; puis par moulinets de 1910 à 1949, ainsi qu'à Nantes mème. Alors que pour le Bas-Rhône et la Garonne, les chiffres ont dû être extrapolés pour les débits dépassant ceux des crues moyennes, les jaugeages au moulinet ont été poussés sur la

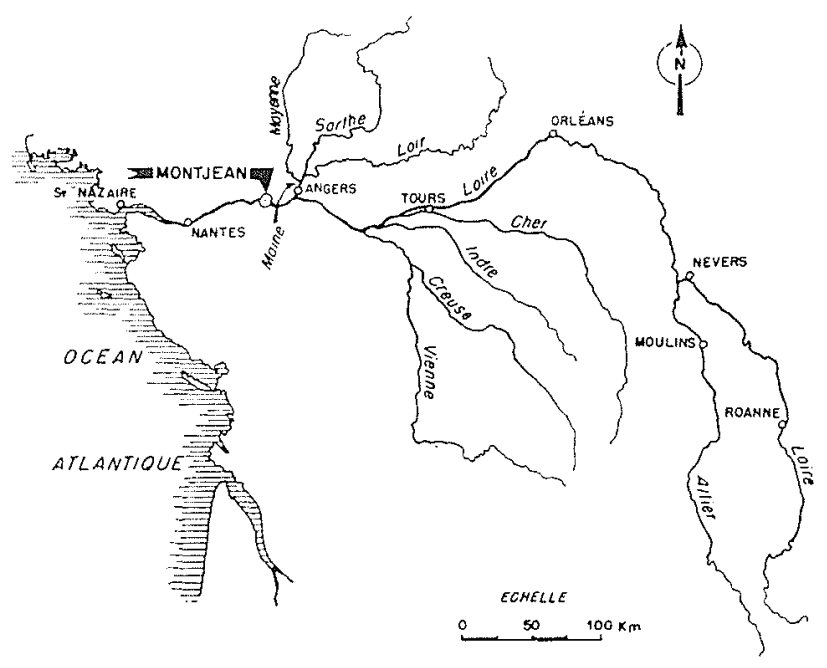

Loire en toutes profondeurs jusqu'à des débits voisins des plus forts maxima connus (1). $\left(6.355 \mathrm{~m}^{3} / \mathrm{s}\right.$ à Montjean en décembre 1910 .)

(1) Les mesures faites au cours des crues de 1910 ont été faites jusqu'au débit de $5500 \mathrm{~m}^{3}$ par l'Ingénieur Nonmandr, qui devait ensuite honorer la Science hydrologique française en dirigeant sur le fleuve Rouge un grand nombre de jaugeages remarquables pour des débits atteignant $20000 \mathrm{~m}^{3}$. 
D'autre part, des mesures nombreuses ont été opérées pour d'autres débits très bien échelonnés et une très grande quantité de mesures ont été faites au voisinage du débit d'étiage. On s'en rend compte d'après les indications fournies sur la courbe des débits utilisée actuellement.

L'idée est venue à M. Pardé, le savant hydrologue de l'Université de Grenoble, d'utiliser ces mesures et la courbe consécutive des débits en fonction des hauteurs à Montjean pour calculer les débits moyens journaliers, puis les moyennes mensuelles et annuelles de chaque année pendant une longue période s'étendant sur 70 années. Ces résultats donneront lieu ultérieurement à d'autres communications de M. PArdé, le présent mémoire s'appliquant avant tout à l'abondance moyenne du fleuve. En l'absence de M. Pardé qui eut été mieux qualifié pour vous les présenter, votre Société a jugé intéressant de me demander d'en établir un résumé, nécessairement imparfait.

\section{Il. - COURBES U'TILISEES}

Jusqu'à il y a une vingtaine d'années, le Service Spécial de la Loire utilisait une courbe de débits à Montjean établie d'après des raisonnements théoriques par l'illustre Ingénieur en Chef SAINJoN à la suite des grandes inondations de 1846,1856 et 1866 . Des courbes de même nature

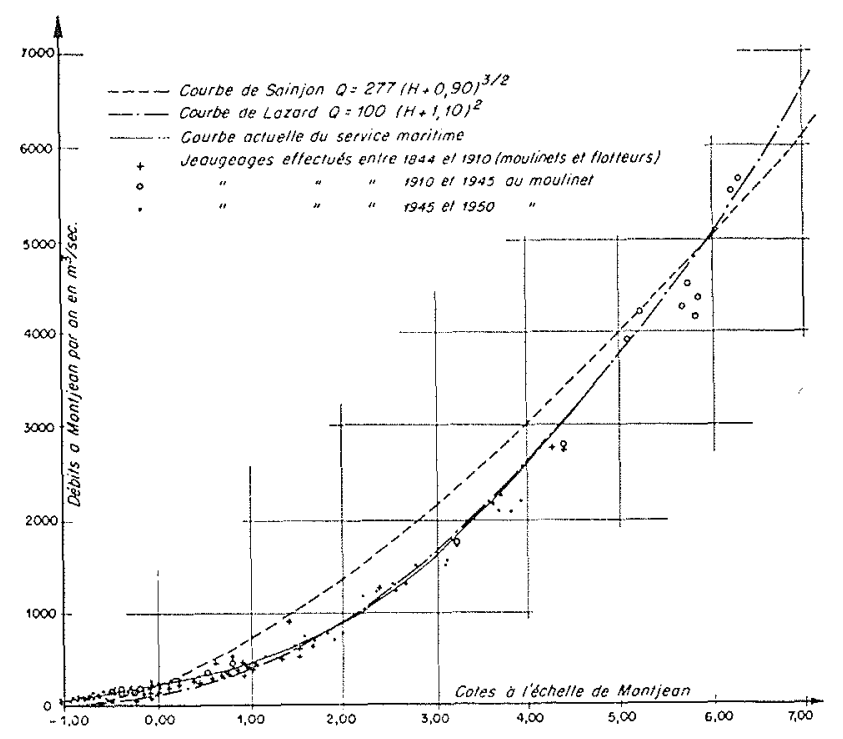

Frg. 1. RÉgime de la Loire a Montuean. Courbes de débits.

avaient été établies d'ailleurs par Sirnson pour les grands affluents de la Loire. Le système de courbes proposé par SaINJon formait un ensemble cohérent qui s'est trouvé assez bien vérifié à
Montjean par les jaugeages ultérieurs, du moins pour les très forts débits.

Néanmoins, comme en 1931 on disposait déjà de nombreux jaugeages, M. l'Ingénieur LAzarD du Service de la Loire à Nantes a fait adopter, sous l'autorité de M. l'Inspecteur Général Notrté, alors Ingénieur en Chef du Service de la Loire à Nantes, une nouvelle formule de débits en fonction de la hauteur à Montjean basée sur l'expérience. Selon l'opinion de M. Pardé, cette courbe serait un peu basse et donnerait des débits un peu trop faibles, parce que d'après lui les résultats des mesures de jaugeages seraient eux-mèmes quelque peu inférieurs à la réalité dans l'ensemble.

Il est de fait que pour la plupart des jaugeages effectués seulement en surface avant 1910 , le coefficient de réduction employé pour en déduire la vitesse moyenne sur une verticale a été seulement de 0,842 (qui est d'ailleurs celui indiqué dans un mémoire de M. Hégly pour déduire la vitesse moyenne générale), alors que d'après des expériences en toutes profondeurs qui ont été faites en Loire il faudrait plutôt compter dans le premier cas sur un coefficient de 0,87 à 0,90 .

M. PARdÉ estime en conséquence que l'emploi de cette courbe a pu conduire pour les modules compris entre 1880 et 1909 à une légère sousestimation de 20 à $40 \mathrm{~m}^{3}$, mais il faut remarquer que la nouvelle courbe ayant servi de base au présent travail représente la synthèse de l'ensemble de tous les jaugeages exécutés au cours des 70 dernières années. Cette nouvelle courbe dite du Service Maritime de Nantes' s'écarte très peu de la courbe LAzARD pour les débits supérieurs à $500 \mathrm{~m}^{3}$.

Une difficulté s'est présentée pour les petits débits inférieurs à 200 ou $300 \mathrm{~m}^{3}$ et surtout pour ceux d'étiage, car les très nombreux jaugeages effectués au voisinage du zéro de l'échelle de Montjean ont montré que ce zéro s'était sensiblement abaissé entre les deux guerres, comme conséquence des travaux d'aménagement à coulrant libre qui ont commencé vers' 1910 entre le confluent de la Maine et le port de Nantes ainsi que des importants travaux d'amélioration du cours maritime de la Loire en aval de Nantes qui ont entraîné une baisse d'étiage importante au port de Nantes (baisse de l'ordre de $1,50 \mathrm{~m}$ ).

Cette dernière cause est d'ailleurs d'après moi la plus importante, l'érosion s'étant développée d'une manière regressive vers l'amont, avec un effet proportionnel à la distance au port de Nantes; la baisse d'étiage entre la Maine et Nantes se présente schématiquement suivant une progression linéaire entre ces deux points.

En définitive, l'étiage à Montjean s'est abaissé d'environ $50 \mathrm{~cm}$ au cours de la période com- 
prise entre 1915 et 1940 sans qu'on puisse dire avec certitude que cet abaissement soit terminé, d'autant plus que même si les anciens travaux ont épuisé leur's elfets, de nouveaux travaux sont projetés (à l'étude sur modèle réduit à Chatou) qui pourront provoquer un nouvel abaissement de l'étiage à Nantes, moins sensible cependant que le précédent.

Le Service de la Loire a été ainsi obligé d'utiliser pour les petits débits de la Loire à Montjean un faisceau de courbes annuelles de débits adaptés à la situation changeante de l'étiage. Pour en donner une idée, alors qu'il $y$ a une vingtaine d'années le zéro de l'échelle de Montjean correspondait à un débit de $100 \mathrm{~m}^{3} / \mathrm{s}$, il donne maintenant le double soit $200 \mathrm{~m}^{3}$.

Pour les hauteurs dépassant $1,50 \mathrm{~m}$ à $2 \mathrm{~m}$ on a admis que les relations des débits aux cotes étaient restées à peu près les mêmes durant les 70 années englobées par les calculs, opinion qui repose sur des présomptions assez fortes basées sur les jaugeages de débits "gros et moyens et sur la considération que le creusement du lit mineur de la Loire dans le cours inférieur a été compensé par les relèvements provoqués dans le lit moyen par les complexes d'épis ayant servi à la régularisation.

Pour ce qui concerne les erreurs rendues possibles en hautes-eaux par des défauts techniques, toujours à craindre en cas de fort courant et d'immersions de moulinets par grandes profondeurs allant jusqu'à 11 mètres, il résulte d'une note détaillée de M. Normandin sur les jaugeages de 1910 en très hautes-eaux que l'erreur totale d'ailleurs incertaine n'a pas dù dépasser 6 à $7 \%$ du débit des crues sans que cet Ingénieur ait pu soupçonner un sens probable à cette erreur.

En définitive, M. Pardé estime que tout compte fait, le module global auquel aboutissent les calculs du Service de la Loire pour les 70 années considérées lui semble susceptible d'une sous-estimation de 20 à $30 \mathrm{~m}^{3}$, sans qu'on puisse affirmer que cette erreur existe vraiment, étant entendu qu'il s'agit plutôt d'une légère erreur par défaut.

\section{III. - VALEUR DU MODULE DURANT LA LONGUE PERIODE DE 70 ANNEES S'ETENDANT DE 1880 A 1949.}

D'après les calculs non rectifiés du Service de la Loire, le module des 70 années s'élève à $840 \mathrm{~m}^{3}$, soit pour un bassin versant de $109.000 \mathrm{~km}^{2}$ à à peu près 7,7 litres par seconde et par kilomètre carré.

En retirant du calcul les 8 années sèches récentes, le module s'élève à $874 \mathrm{~m}^{3}$ alors que pour les 50 années à partir de 1880 on n'obtiendrait plus que $801 \mathrm{~m}^{3}$, ce qui révèle de fortes fluctuations au cours des groupes d'années.

Quoi qu'il en soit, le module est compris entre 800 et $900 \mathrm{~m}^{3}$ pour les 50 à 75 années les plus récentes, ce qui est le double de la valeur communément citée il y a encore un quart de siècle,

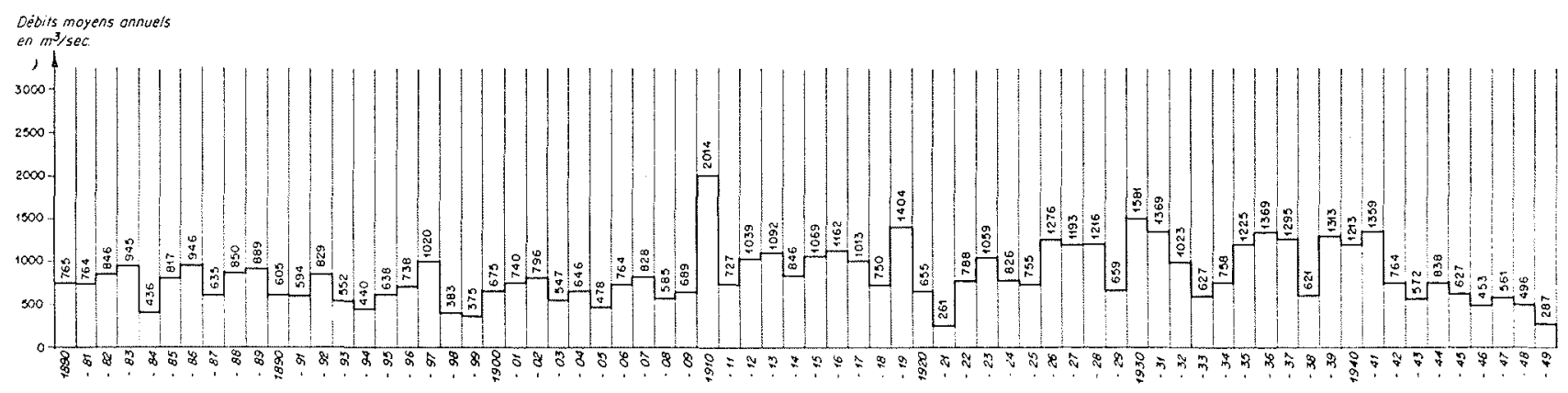

Fig. 2. - Étude du régme de la Lorre a Montuean. Graphique récapitulatif des débits moyens annuels.

bien que M. PARDÉ dans son petit livre «Fleuves et Rivières » publié en 1933 ait déjà indiqué pour le module de la Loire une valeur probable voisine de celle qui vient d'être déterminée. Et cependant M. PARDÉ ne disposait pas encore des résultats d'expériences, et avait simplement basé son opinion sur les idées très cohérentes qu'il avait réunies concernant les facteurs principaux et les valeurs régionales probables des coefficients ou quotients annuels d'écoulement (alors que plus tard on a pris de préférence pour base des évaluations, les déficits d'écoulement).

Toutes ces considérations dues à la sagacité de M. PArdé lui avaient permis d'avancer dès 1925 dans l' « Encyclopédia Italiana » un module brut de $933 \mathrm{~m}^{3}$ pour un bassin versant total de $120.000 \mathrm{~km}^{2}$ (moyenne pondérée de $740 \mathrm{~mm}$ pour les précipitations annuelles et coefficients d'écoulement de l'ordre de $33 \%$ ). Le travail de M. PARdÉ, qui s'est d'ailleurs étendu 
à tous les afluents de la Loire, est condensé dans un tableau joint au mémoire original (1).

M. PARó́ fait d'ailleurs remarquer que les évaluations géographiques de ce genre, appliquéc à des bassins fluviaux assez grands pour que les anomalies locales du bilan d'écoulement pèsent

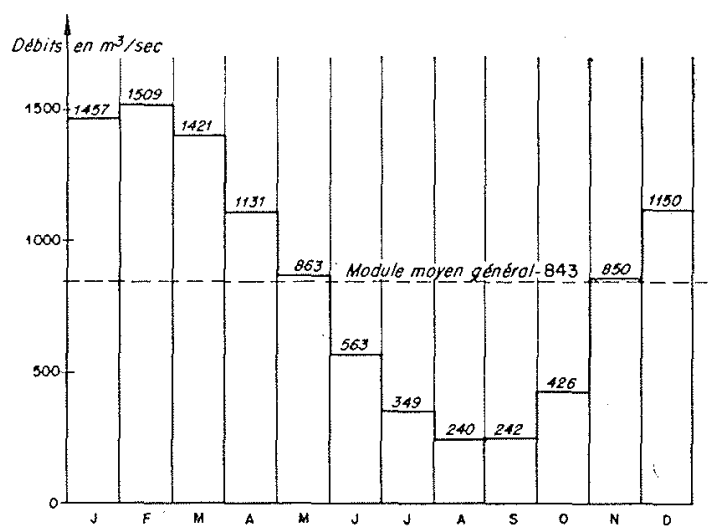

Hia, 3. - Débits Moyexs Miensueds de la Loire a MONTAEAN POUR LA PERIODE 1880-1949.

d'un faible poids sur les débits totaux, donnent des résultats cohérents pour l'hydrologue qui a la pratique des bilans annuels de l'écoulement dans les pays tempérés. Ceci ne diminue d'ailleurs en rien le mérite de M. Pardé qui manie ses données avec beaucoup d'habileté. Il est remarquable en effet que les résultats d'expériences pour le module à Montjean, compte tenu de la légère sous-estimation qui a pu ètre donnée par les jaugeages, concordent très exactement avec l'approximation ancienne de $M$. PARDÉ basée sur des considérations générales.

Si l'on veut obtenir d'autre part le module de la Loire à son embouchure il faut tenir compte de ce qu'entre Montjean et cette embouchure la surface réceptrice s'accroìt de $8.000 \mathrm{~km}^{2}$ environ pour laquelle on dispose des relevés pluviométriques de M. Angot pour la période 18511900 et de M. J. SAnson pour la période de 18801930 (température moyenne annuelle voisine de $11^{\circ} 5$ ). M. Parnê calcule ainsi que le module à l'embouchure doit être estimé à $900 \mathrm{~m}^{3}$, avec une grande probabilité, pour les 70 années considérées.

Il est intéressant de remarquer que ce module est la moitié de celui du Bas-Rhône, mais reste supérieur à celui de la Garonne à Bordeaux (compris entre 630 et $660 \mathrm{~m}^{3}$ ), supérieur aussi à celui de la Seine qui est de l'ordre de $500 \mathrm{~m}^{3}$. Par contre on observe plus de $1.000 \mathrm{~m}^{3}$ pour le Rhin à Bâle, $1.500 \mathrm{~m}^{3}$ pour le Pồ et les modules suivants pour d'autres rivières : Douro $650 \mathrm{~m}^{3}$ à

(1) Mémoire versé in extenso aux archives de la Société.
$675 \mathrm{~m}^{3}$, Tage $450 \mathrm{~m}^{*}$ à $475 \mathrm{~m}^{3}$, Elbe $675 \mathrm{ml}^{3}$, Oder $500 \mathrm{~m}^{3}$, Meuse $280 \mathrm{~m}^{3}$, Adour $350 \mathrm{~m}^{3}$, etc... La Vistule, avec $950 \mathrm{~m}^{3}$ au moins, n'est guère plus abondante que la Loire.

Par contre le module de la Loire diminue rapidement quand on remonte son cours. On notera en effet qu'à 'Tours qui n'est pas très loin de l'embouchure, la Loire, malgré son large lit, n'est guère plus abondante que la Seine dans la capitale avec 360 à $380 \mathrm{~m}^{3}$ contre 310 à $320 \mathrm{~m}^{3}$. Cela tient à la conformation peu harmonieuse du réseau de la Loire qui amene plus de la moitié du débit total à partir du confluent avec le Cher'.

Pour le module spécifique la Loire avec 7,7 à $8 \mathrm{l} / \mathrm{s}$ par $\mathrm{km}^{2}$ l'emporte sur la Seine, 6,05 à 6,35; et encore plus sur la Vistule 5,7, l'Elbe 5, l'Oder 4,7, l'Ebre 7,4, le Douro 6,7, le Tage 5,6; mais elle est battue à cause des fortes précipitations pyrénéennes par la Garonne 11,5 à 12 et plus encore à cause de la pluviosité alpestre par le Rhin (15), le Rhône (19) et surtout par le Pô (24). Le Rhin à Bâle donne environ 28 .

\section{IV. - BILAN ANNUEL DE L'ECOULEMENT}

Le module faisant ressortir une hauteur d'environ $250 \mathrm{~mm}$ pour la pluie écoulée contre $750 \mathrm{~mm}$ de précipitation moyenne approximative, il en résulte que pour l'ensemble du bassin le déficit annuel d'écoulement est d'environ $500 \mathrm{~mm}$. Ce déficit d'écoulement est tout à fail du même ordre de grandeur que celui qu'on peut trouver pour la Seine, pour la Garonne et pour le Rhône, alors que les modules spécifiques révèlent les différences considérables qui ont été indiquées plus haut.

Cette quasi-similitude des pertes en eau atmosphérique pour de grands bassins fluviaux d'une même région étendue entre plusieurs degrés de latitude est maintenant un des axiomes de l'hydrologie fluviale. Il résulte du fait que plus une surface réceptrice est vaste, plus les anomalies positives ou négatives locales par rapport à la moyenne régionale tendent à se compenser les unes par les autres.

Pour de petits bassins versants de 50 à $100 \mathrm{~km}^{2}$ dans les Hautes-Alpes, les déficits peuvent ne pas dépasser $200 \mathrm{~mm}$ alors que dans les Landes ils dépassent $600 \mathrm{~mm}$.

Ces faits ont été mis en évidence par les belles études des professeurs allemands H. KaELLER, K. Fischer et M. Wundt, puis par l'éminent Ingénieur français A. Coutagne et souvent aussi, dois-je le dire, par M. Pardé lui-même.

Rappelons que cette constatation a conduit les hydrologues à prendre de préférence comme 
bases de leurs évaluations sur les modules ou de leurs commentaires sur les bilans d'écoulements, les déficits plutòt que les coefficienls d'écoulements. Ceci ne veut pas dire, au contraire, que les inégalités de ceux-ci n'aient pas une signification géographique de premier ordre.

Indiquons également d'après M. Parbŕ que te déficit d'écoulement qui vient d'être proposi pour la Loire ne paraît guère supérienr aux valeurs généralement admises pour l'Europe occidentale (1).

Il semble toutefois que le déficit discuté pour la Loire l'emporte de 30 à $40 \mathrm{~mm}$ sur la normale correspondant aux mêmes pluies et à ha température moyenne probable qui est nécessairement abaissée (sans doute à moins de 10") par le haut-relief d'une partie notable du bassin, celle où tombent les plus grosses précipitations.

M. PARDÉ précise qu'il faut trouver les raisons de laugmentation du déficil dans le bassin supérieur de la Loire, c'est-ä-dire en amont du Bec d'Allier, dans le caractère déjà continental des pluies sur celte région, c'est-à-dire dans une proportion très forte des chutes d'eau estivales, les plus attaquées de beaucoup par l'évaporation et par les besoins des plantes. C'est à cause do ce facteur, encore plus marqué à l'est du Rhin, qu'on trouve pour les principaux fieuves d'Allemagne et de Pologne des déficits qui dépassent les valeurs qu'on anrait dans le bassin de Paris pour des pluies et des températures respectivement analogues. Enfin l'irrigation de zones étendues de prairies par un nombre très grand de petits canaux consomme une quantité d'au qui ne revient pas à la rivière.

\section{r. - FLUCTUATIONS DE L'ABONDANCE MOYENNE}

\section{RAPPORTS DES MODULES EXTRÊMES}

Il itait admis précédemment d'après la régularité du climat tempéré non méditerranéen de nos régions que le rapport du module le plus fort au débit moyen annuel le plus faible ne devait guère dépasser 4 à 5 pour les rivières du type pluvial océanique européen comme l'est en somme le cours inférieur de la Loire, ou du type pluvionival également océanique, régime auquel obéissent les tronçons hydrographiques supé-

(1) Voir Je conrs de M. Parbé : Polamologie ", polycopic a l'Ecole des Ingénieurs Hydrauliciens de l'Université de Grenoble, 2 vol., 1949, pages $159-177$ et, du mème auteur, « les Facteurs géographiques du bilan annuel de l'écoulement fluvial $\%$, dans la revue hongroise Hydrologiai Közlöny, 1949, fascicules 5-6. rieurs dans les bassins de l'Allier et de la HauleLoire.

Si l'on compare le module de $2.014 \mathrm{~m}^{3}$ atteint en 1910 à celui de 1921 qui s'est abaissé à $255 \mathrm{~m}$;, le rapport est de 7,9 soit 8 environ, chifTre qui fait bien ressortir l'instabilité des débits gouvernés par nos climats. Le module de 1948 . 49 s'est mème abaissé à $244 \mathrm{ml}^{\prime 3}$ et le rapport des modules extrênes connus monte alors à 8,25 .

Pour savoir à quelles fréquences (par exemple $1 / 1.000^{\circ}$ ou $2 / 1.000^{\circ}$ ou $5 / 1.000^{\circ}$ ) correspondent ces modules anormaux, la seule ressource est de recourir au calcul des probabilités en prenant la liste brute on légèrement rectifiée des modules de 70 ans pour base.

Sans dénier de l'intérêt à ces recherches d'ordre mathématique, M. PArdé pense, et il it souvent exprimé cette opinion, que les calculs de ce senre ne peuvent etre accueillis qu'arec réserves jusqu’à rérification ultérieure par les faits, vérification d'ailleurs plus théorique que pratique, car pour ètre fondée, elle devrait résulter d'observations étendues à des périodes très longues : par exemple 1.000 ans pour les modules dont lintervalle serait de 100 ans, 10.000 ans pour les modules espacés en moyenne de 1.000 ans et lorsque le nombre indispensable de siccles ou de millénaires se serait écoulé, la tendance fondamentale du climat aurat pu changer assez pour modifier l'hydrologie de nos fleuves. A tout hasard il parait vaisemblable que lo débit moyen annuel à Montjean puisse dans lintervalle moyen de 500 à 1.000 ans atteindre jusqu'à $2.500 \mathrm{~m}^{3}$ on s'effondrer à 200 ou mème $175 \mathrm{~m}^{3}$.

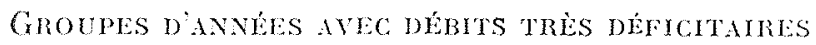

Il avait semblé jusqu'à présent à M. PARDÉ que pour des rivières comme la Seine, la Tamise, la Loire, etc..., les groupes d'années de $\&$ a 10 ans caractérisés par les débits les plus maigres, ne pouvaient guère connaitre un déficit plus srave que de 12 a $15 \%$ par rapport à la moyenne de 50 à 100 ans.

Or pour la Loire cette hypothèse est infirméc car les $\&$ années qui s'étendent de 1942 à 1949) correspondent à un déficit de $31,5 \%$ par rapport à $840 \mathrm{~m}^{\prime 3}$, chiffre adopté comme la valeur normale d'une longue période à Montjean.

Des indices concordant de plus en plus nombreux semblent révéler que la faiblesse néfaste des débits de la période 1942-1949 s'est étendue a l'Italie, aux bassins de la Volga, à l'Afrique du Sud, à l'Amérique du Sud, à la NonvelleZélande et à une partie de l'Australic. On sest demandé si ces phénomènes ne révélaient pas une tendance à un appaurissement hydrolosicque susceptible de s'accentuer d'une manière catastrophique dans les siècles prochains. Sans 
que la possibilité du fait puisse être contestée, il est aussi bien permis d'espérer qu’il s'agit seulement d une anomalie négative plus profonde que beaucoup de ses devancières, d'ailleurs mal ou point chiffrable, et que dans un avenir plus ou moins proche, une tendance inverse se manifestera. Dans un fascicule de la Revue de Geographie Aipine paru en 1950, M. P'andé a prosenté sur ce sujet, en collaboration avec M. SAxsox, des arguments assez contradictoires, mais finalement assez rassurants.

Pour en revenir à la Loire, il résulte de l'examen des modules suceessifs à Montjean que co fleuve a élé beaucoup moins alimenté dans l'ensemble de 1880 à 1909 que de 1910 a 1941 avant la pénurie plus récente déplorée plus haut.

Dans la moyenne de 30 ans (1880-1909) ie fleuve a débité seulement $694 \mathrm{~m}^{3}$ soit un écart négatif de $17,5 \%$ et mème les 20 ans les plus pauvres (1890-1909) accusent avec $646111^{3}$ une insuffisance extraordinaire pour 20 années de $23 \%$, qui serait portée à $26,5 \%$ pour la seule période 1890-1899.

En revanche les 32 annes qui ont commenci en 1910 montrent pour la Loire une surabondance très sensible. Malgré l'efTet déprimant des pénuries de 1920 et de 1921 le module de ces 32 années dépasse $1.027 \mathrm{~m}^{3}$ soit un excédent de $22 \%$ qui deviendrait $24 \%$ pour 32 ans. $(1.043 \mathrm{~m}:$ pour la période 1910-1941).

Mème en faisant la part des erreurs possibles sur les jaugeages il ne semble guère possible de contester qu'en 10 ou 12 ans la Loire à Montjean puisse rouler des débits supérieurs de 25 à $30 \%$ à la normale d'une longue suite d'annces.

M. PARDÉ ne connail point des cas semblables pour des rivières francaises et d'Europe centrale ou orientale. Il existe done une sensibilité particulierement vive de la Loire aux variations des pluies.

M. Pardé a également recherché s'il était possible de constituer des groupements d'années d'abondance ou de pénurie.

Bien qu'il existe une tendance des modules maigres ou surabondants à se grouper en suites d'années plus ou moins longues, ces groupements comportent trop d'exeptions pour qu'it soit possible d'en déduire des lois de proviodicité utilisables dans la pratique.

\section{V1. - AUTRES ELEMENTS DU REGIME}

\section{VARITIONS SAISONNIERES MOYENNES}

Le régime des 70 ans appartient nettement au týpe pluvial océanique par son maximum de février, par la suprématie du semestre décembremai qui correspond arec un retard de 1 mois a
1 mois et demi à la saison froide, et par les basses-eaux de fin d'été. Il est rappelé que cellesci ont pour cause non la médiocrité des pluies estivales (on observe plutôt le contraire dans l'ensemble du bassin), mais l'activité intense de l'éraporation et de la transpiration par les plantes. Le maximum si marqué de janvier-mars est dù à la faiblesse de l'évaporation et non à une supériorité inexistante des chutes pluviales en celte saison.

Le mois de mars pouvant correspondre au maximum lorsque des précipitations importantes concordent avec un temps de redoux qui cntraine des fontes abondantes de neige dans le Massif Central, cette dernière cause a certainement contribué à rendre les débits de mars supérieurs à ceux de janvier de 1880 à 1909 , ce qui permet de dire que le régime n'est point pluvial pur, mais quelque peu pluvio-nival.

\section{EThages extrêmes}

Les pénuries graves selon les règles du régime pluvial surviennent presque toutes en été, au début de l'automne quand les pluies attendues a cette époque manquent ou sont insuffisantes. A Montjean les plus faibles débits connus ont été observés en septembre un peu plus fréquemment qu'en août. Le record d'indigence a étó constaté avec $48 \mathrm{~m}^{3}$ en août 1949, module qui correspond à un ćcoulement spécifique do $0,44 \mathrm{l} / \mathrm{s}$ par $\mathrm{km}^{2}$. On peut le comparer avec les minima minimorum rarement inférieurs à 5 ou $6 \mathrm{l} / \mathrm{s}$ par $\mathrm{km}^{2}$ qu'on observe sur les cours d'eau issus des hauts massifs pyrénéens ou des Grandes Alpes.

Par contre la Seine, malgré sa réputation de riviore pondéré, semble autant que la Loire capable d'un débit naturel inféricur à $0,5 \mathrm{l} / \mathrm{s}$ par $\mathrm{km}^{2}$.

Lors des basses-eaux d'aout 1949, la Loire a cté jaugée en amont puis en aval du confluent avec la Maine et ces mesures ont révélé que le débit de l'aflluent était pratiquement réduit à rien. Ce phénomène par comparaison laisse juger possible de tres faibles étiages sur la Seine qui auraient été autrefois considérés comme irréalisables.

D'après l'Ingénieur en Chef d'Orléans le débit de la Loire n'aurait pas dépassé $12 \mathrm{~m}^{3}$ au mois de juin au cours de l'été 1949.

\section{Crues}

Les crues de la Loire constituent un véritable monde de phénomènes nécessitant une étude d'une ampleur considérable.

Il est possible, sinon probable que le maximum de $6.355 \mathrm{~m}^{\circ}$ de 1910 soit le plus fort éprouvé dans le cours inférieur de la Loire depuis jan- 
vier 1843 exclusivement. Encore les ruptures des digues de Saint-Georges et de Montjean ont-elles quelque peu déprimé les pointes qui sans cet accident auraient avoisiné 6.500 à $6.700 \mathrm{~m}^{3}$. Le maximum de la fameuse crue de mai-juin 1856, qui a rompu la grande levée d'Anjou n'a probablement pas égalé le chiffre de décembre 1910 (1) à Montjean, mais celui-ci a peut-être été dépassé ou tout au moins égalé en janvier 1843, sans certitude toutefois car cette dernière crue, plus forte à Saumur qu'en 1910, a pu être moins renlorcée par la Maine et par les affluents mineurs.

Il est à remarquer que les crues de 1843 , de 1910, mars 1923 et janvier 1936 ainsi que la plupart des maxima supérieur's à $5.000 \mathrm{~m}^{3}$ à Montjean et à Nantes sont du type océanique pur et n'ont présenté aucune gravité sur le fleuve en amont du confluent avec la Vienne dont I'apport est très important.

Bien plus remarquables et dévastateurs sur l'ensemble du val de Loire et à l'amont sont les cataclysmes d'origine plus ou moins méditerrancenne et fort probablement mixte (à la fois méditerranéenne et atlantique); ils occasionnent de beaucoup les plus hauts niveaux connus à Roanne, Moulins, puis au Bec d'Allier et en aval jusqu'au confluent de la Vienne ou de la Maine.

Les seules grandes crues de celte espèce qu'on ait observé après 1866 furent : celle d'octobre 1872 qui donna $4.300 \mathrm{~m}^{3}$ à Orléans et peut-être 3.500 à Montjean, puis celle d'octobre 1907 qui fut plus grave en amont du Bec d'Allier.

Depuis une quarantaine d'années les crues mćditerranéennes ou mixtes de la Loire n'ont eu aucune gravité, mais auparavant en octobre 1846, mai-juin 1856 et septembre 1866 trois déluges de ce genre se succédèrent à des intervalles de 10 ans. Leurs débits au Bec d'Allier ont été à peu près doubles de ceux d'octobre 1872 et de 1907, peut-être triples ou quadruples de ceux des crues océaniques indiquées plus haut. (Au Bec d'Allier, débit de $9.800 \mathrm{~m}^{3}$ en $1846,8.800 \mathrm{~m}^{3}$ en 1856 et $9.800 \mathrm{~m}^{3}$ en 1866$)$. Mais en aval, de nombreuses et terrifiantes

(1) La grande levée royale d'Anjou, qui protège 40000 hectares des riches terres du val de l'Authion sur la rive droite de la Loire depuis Langeais jusqu'à Angers, peut faire remonter son origine jusqu'au roi Henri II d'Ausleterre de la dynastie des Plantagenets, qui, comme Comte d'Anjou, mit sur pied une organisation de défense contre les crues, dont les traces subsistent encore. Ce haut seignear, intéressé au défrichement et à la mise en valeur de cette région, eut l'idée géniale d'installer de distance en distance sur la première levée des sortes de colons, en même temps mi-soldats, bénéficiant de nonbreuses exemptions, mais tenus de velller à la conservation des ouvages en temps de menaces du fleuve. Ces centres de colonisation militaire sont devenus aujourdhui des villages qui ornent la levée d'Anjou : SaintMartin-de-la-Place, Saint-Lambert-des-Levées, Saint-Mathurin, etc. ruptures de digues et d'énormes submersions puis la médiocrité ou l'avance des renforts apportés par les affluents du cours inférieur ont fait que sur celui-ci, à partir du confluent avec la Maine, les maxima de 1846 et 1866 n'ont plus égalé ceux de maintes crues océaniques pures formées sur la partie occidentale du bassin. Les débits' ne dépassèrent point à Montjean $4.500 \mathrm{~m}^{3}$ en 1846 , ni $5.200 \mathrm{~m}^{3}$ en 1866 . Seule la crue de mai-juin 1856 a rompu à peu près toutes les digues jusqu'à Nantes et rivalisẻ avec les inondations atlantiques de janvier 1843 et décembre 1910. Elle a dû débiter environ $6.000 \mathrm{~m}^{3}$ vers Montjean. Certains indices peuvent laisser le soupçon que la crue visiblement océanique de février 1711, a, sur le cours inférieur, roulé des débits sensiblement plus forts que ceux de 1843 et ceux de 1910, mais c'est une impression plus qu'une probabilité.

Enfin on ne connaît pas d'exemple d'une coïncidence entre un maximum plus ou moins méditerranéen très fort arrivant du Bec d'Allier et un grand flot océanique élaboré quelques jours après l'averse génératrice de la crue méditerranéenne. Une telle superposition, qui ne semble pas du tout impossible, produirait dans le bassin de la Loire et notamment à partir du confluent avec la Vienne des maxima tout à fait destructeurs.

$$
\text { \% }
$$

\section{CONCLUSION}

Le sujet a été à peine effleuré. Son importance est grande, car le bassin de la Loire couvre le quart de notre territoire et son régime commande la vie d'une nombreuse population. Heureusement M. PARDÉ nous promet de nouvelles communications, que votre Société accueillera sans aucun doute avec un grand intérêt.

Puis-je me permettre de signaler que le Service de la Loire considère avec attention tous les projets, ayant en vue de modifier le régime si capricieux du fleuve. Je fais ainsi allusion au projet de captage des eaux souterraines du val de Loire pour l'agglomération parisienne, ainsi qu'à ceux ayant pour objet d'emmagasiner les caux du bassin supérieur pour les restituer non à la Loire, mais' à un autre bassin méditerranéen, dans le seul but d'en tirer des ressources supplémentaires d'énergie hydro-électrique.

Selon la manière dont de tels travaux peuvent être conçus, ils peuvent être désastreux ou avantageux pour les riverains de la Loire. Il ne faut pas oublier que ceux-ci ont besoin avant toute chose de cette eau, et non pas des kilowatt- 
heures créés par son détournement. Sans doute l'eau de la Loire est-elle surabondante et dévastatrice pendant les crues dépassant un certain niveau. Mais les crues moyennes fertilisent les terres et alimentent les eaux des nappes; les eaux basses doivent aussi être assez soutenues pour permettre à la navigation de s'exercer dans le cours inférieur, et leur trop bas niveau engendre des troubles dans la partic maritime.

Si donc on ne peut parler d'un danger réel à l'occasion du soutirage modeste que comporte le chantier de Montpézat (ayant pour effet de détourner dans le bassin de l'Ardèche une petite partie des eaux de la Loire près de sa source), la prudence s'impose dès qu’il s'agit de grandioses projets d'emmagasinement portant sur plusieurs milliards de mètres cubes, destinés non à être restitués à la Loire, mais à être détournés sur le Rhône ou vers Béziers.

Il est nécessaire qu'en pareille hypothèse le souci d'accroître nos ressources énergétiques soit subordonné au résultat d'une étude plus large, ayant pour objet d'améliorer le régime de la
Loire, en ayant présent à l'esprit les intérêts de l'agriculture, de la navigation et du port de Nantes. Il n'est pas interdit de penser qu'on puisse s'inspirer des' grands travaux effectués en Allemagne pour la régularisation du régime de l'Elbe, au moyen de barrages sur le cours supérieur, qui ont eu pour effet d'emmagasiner des volumes d'eau excédentaires en hiver et de les restituer en été pour soutenir le niveau d'étiage.

Nous formons le vou, et ce sera notre conclusion, que, le moment venu, le régime du cours inférieur de la Loire puisse être amélioré à l'occasion des modifications qui seront éventuellement apportées dans son bassin supérieur du Massif Central.

\section{COMMENTAIRE DE M. LE PRÉSIDENT}

M. Io Président interprète le sentiment unanime du Comité Technique en félicitant MM. Gibert et Pardé pour lenr communication, qui est riche, non seulement de précisions, mais aussi de vues d'ensemble qui peuvent permettre de réflechir et de chercher des solutions aux problèmes posés.

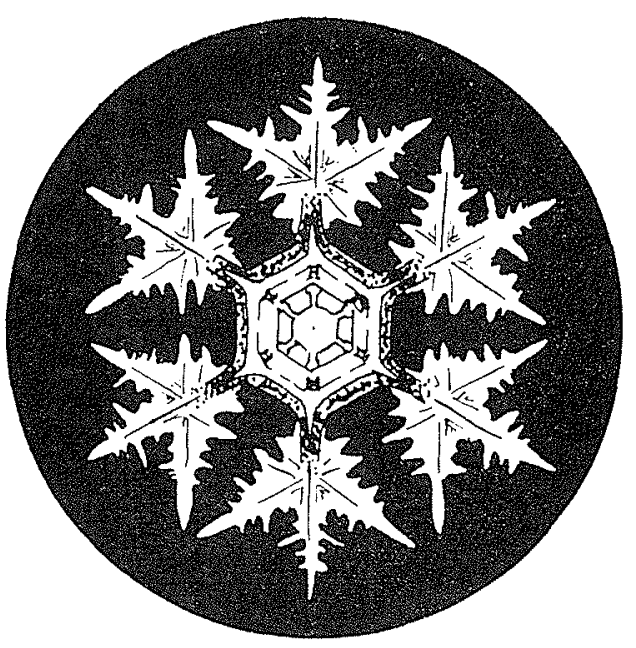

